\title{
Utilisation of paediatric surgical theatres at the Chris Hani Baragwanath Academic Hospital, Johannesburg
}

S Ford, ${ }^{1} 6^{\text {th }}$-year medical student; N Brink, ${ }^{1} 6^{\text {th }}$-year medical student; $\mathbf{N}$ Martin, ${ }^{1} 6^{\text {th }}$-year medical student; S Soares, ${ }^{1} 6^{\text {th }}$-year medical student; B Manicom, ${ }^{1} 6^{\text {th }}$-year medical student; T Mahadea, ${ }^{1} 6^{\text {th }}$-year medical student; $\mathbf{M}$ Reynolds, ${ }^{1} 6^{\text {th }}$-year medical student;

A Grieve, ${ }^{2}$ FCPaed Surg (SA); J Loveland, ${ }^{2}$ Cert Paed Surg (SA); T Gabler, ${ }^{2}$ FCPaed Surg (SA)

${ }^{1}$ Faculty of Health Sciences, University of the Witwatersrand, Johannesburg, South Africa

${ }^{2}$ Department of Paediatric Surgery, Faculty of Health Sciences, University of the Witwatersrand, Johannesburg, South Africa

\author{
Corresponding author: S Ford (sarahford711@gmail.com)
}

\begin{abstract}
Background. Optimal theatre utilisation is essential to reduce long waiting lists for elective surgeries and to increase cost-effective theatre operation. Utilisation rates well below the global benchmark of $80 \%$ have been reported for government hospitals in Johannesburg, South Africa (SA).

Objective. To investigate utilisation of three paediatric theatres at the Chris Hani Baragwanath Academic Hospital (CHBAH) in Johannesburg.

Methods. Surgery lists of the three theatres were reviewed for three one-week periods over a four-month study period. Preoperative, intraoperative and postoperative data were collected to create a timeline for each theatre and each surgical case, including reasons for cancellations, delays or expedited times.

Results. A total of 152 surgeries were scheduled during the reviewed study period, of which 44 cases were cancelled. The utilisation rate was $59.8 \%$ across the three theatres combined, with individual rates calculated as $62.7 \%, 58.2 \%$ and $57.0 \%$ in the burns, general and neonatal theatres, respectively. The primary factor contributing to under-utilisation was early completion of the scheduled list. Surgery delays were mainly due to delays in transferring the patient to the theatre and between anaesthetic induction and the start of surgery. Conclusion. Utilisation of the paediatric theatres at CHBAH is below the ideal benchmark of $80 \%$; however, utilisation was better than expected when compared with findings from other public-sector hospitals in SA. However, theatre efficiency was found to be very low and perhaps better explains the reasons for backlogs in paediatric surgeries at $\mathrm{CHBAH}$.
\end{abstract}

S Afr J Child Health 2021;15(4):185-188. https://doi.org/10.7196/SAJCH.2021.v15i4.1774

Scheduled elective surgeries are increasing in the public health sector in Gauteng province, South Africa (SA), with the waiting list for these procedures consequently extending. Prior to the COVID-19 pandemic there were 200 cases on the waiting list in the Paediatric Surgery Pepartment at Chris Hani Baragwanath Academic Hospital (CHBAH). (The backlog in elective surgeries has subsequently increased considerably owing to pressure on healthcare facilities during the ongoing pandemic.) Efficient use and scheduling of operating theatres are essential to reduce waiting lists and improve patient outcomes.

In the SA context, where public healthcare is constrained by both limited funds and staff, it is imperative to optimise theatre utilisation to alleviate the pressure on these resources. ${ }^{[1]}$ Theatres are expensive to run, even when not in use. Better theatre utilisation can, therefore, contribute to improved cost-effectiveness of surgical departments and hospitals.

Theatre utilisation is defined as the ratio between time spent operating and the daily available theatre time. (Theatre time is defined as the period during which a theatre is available for operations, usually from 08:00 to 16:00. The cut-off time for the last patient having to leave the operating theatre is 16:00.) A utilisation ratio of $80 \%$ is accepted as ideal across the world, and takes into account unavoidable parameters such as theatre sterilisation and patient preparation.
A study describing theatre utilisation in a private setting in SA has shown that the theatre complex ran at less than half the optimal rate, despite being privately funded $;^{[1]}$ another at a public hospital in North West Province, SA, showed that theatres were similarly underutilised, with an average utilisation rate of $39 \% .{ }^{[2]}$ These studies suggest that theatre utilisation rates are likely comparable in the public and private sector in SA, and well below the ideal benchmark in both settings.

This prospective study investigated theatre utilisation in three units of the Paediatric Surgery Department at CHBAH. Identifying factors that affect the use of paediatric operating theatres can be useful in understanding how theatre utilisation and efficiency can be improved and, in turn, shorten the waiting list for pending elective surgeries.

\section{Methods}

This was a prospective study conducted in the paediatric burns theatre, the general paediatric theatre and the neonatal theatre of $\mathrm{CHBAH}$ in Johannesburg, SA. These theatres handle neonatal emergencies and general elective paediatric surgeries. All elective procedures were included in the data collection process. The study was performed over a cumulative three-week period between April and July 2019. 


\section{Data handling}

Three sets of data were collected for each case (preoperatively, intraoperatively and postoperatively) and entered into a database system (REDCap). Preoperative data included: records of any cancellations, the expected duration of a procedure, the time at which the patient was collected from the ward and the time when the patient arrived in theatre.

Intraoperative data included the time stamp of the anaesthetist's and surgeon's arrival, the time of anaesthetic induction, start and end times of the surgery, and the time at which the patient left the theatre.

Postoperative data included: the time at which the patient was admitted to the recovery unit, their length of stay, and the time at which they arrived back at the ward. At each point of the data collection process, it was noted whether documented times were appropriate and if not, a reason was provided.

Data were collected for a total of three weeks over a four-month period. Documented times were used to calculate descriptive statistics (mean, standard deviation, median and mode) and interquartile ranges (IQRs) were calculated for each time interval.

The duration between the start of anaesthetic induction and when the patient left the theatre was totalled for each theatre list. Total values for each list were then divided by the total available time for that list (8 hours; from 08:00 to 16:00) to express theatre utilisation as a percentage. Average durations per theatre and also for all three theatres combined were subsequently calculated.

Efficiency was calculated according to Pandit et al. ${ }^{[3]}$ as: (Utilisation - Fraction of scheduled time overrunning) $\times$ Fraction of scheduled operations completed.

\section{Ethical considerations}

Ethical approval for the study was obtained from the Human Ethics Research Committee of the Faculty of Health Sciences, University of the Witwatersrand (ref. no. M180941).

\section{Results}

There were 152 surgeries scheduled in the three weeks of data collection, of which 71 were in the neonatal theatre, 50 in the general theatre and 31 in the burns theatre. Of these surgeries, 147 were elective procedures and 5 were emergencies. Slightly more than a quarter of the scheduled surgeries ( $n / N=44 / 152 ; 29 \%)$ were cancelled ( 25 from the neonatal theatre; 14 from the general theatre; 5 from the burns theatre). The two most common reasons for cancellation were that the patient did not show up and time constraints (Fig. 1a). Specific reasons for cancellation in the respective theatres are shown in Fig. 1b.

Of the 27 lists run during the data collection period, only seven were completed as booked; the other 20 had at least one cancellation.

Fig. 2 shows the average time for each step in the normal theatre process, both for each theatre individually and for the complex as a whole.

Table 1 shows the most common reasons for the delays between recorded points and the percentage of cases affected by these delays. In general, the neonatal theatre had the latest start time, and the burns theatre the earliest (See Appendix 1 for the specific start times of lists in the respective theatres on each day of data collection.) The median start and end times for the theatre complex were 08:36 (IQR: $38 \mathrm{~min}$ ) and 15:17 (IQR: $2 \mathrm{~h} 13 \mathrm{~min}$ ), respectively. The median end times for lists were 14:09 (IQR: $3 \mathrm{~h} 14 \mathrm{~min}$ ), 15:10 (IQR: $2 \mathrm{~h} 15 \mathrm{~min}$ ) and 15:36 (IQR: 57 min) for the general, burns and neonatal theatres, respectively. A third of the 27 lists $(n=9)$ reviewed during the data collection period ended late (i.e. after 16:00); of these, five were from the neonatal theatre, two from the general theatre and two from the burns theatre.

The general utilisation percentage at the $\mathrm{CHBAH}$ paediatric theatre complex was $59.8 \%$. Utilisation rates for the specific

Table 1. Surgery delays reported for theatres of the paediatric theatre complex at Chris Hani Baragwanath Academic Hospital, Johannesburg

\begin{tabular}{ll}
\hline Reason for delays & Cases \\
\hline Patient called late & 25 \\
Patient arrived at theatre reception late & 37 \\
Patient arrived in theatre late & 29 \\
Delay between anaesthetic induction and surgical start & 37 \\
Surgeon arrived late & 19 \\
Anaesthetist arrived late & 7
\end{tabular}

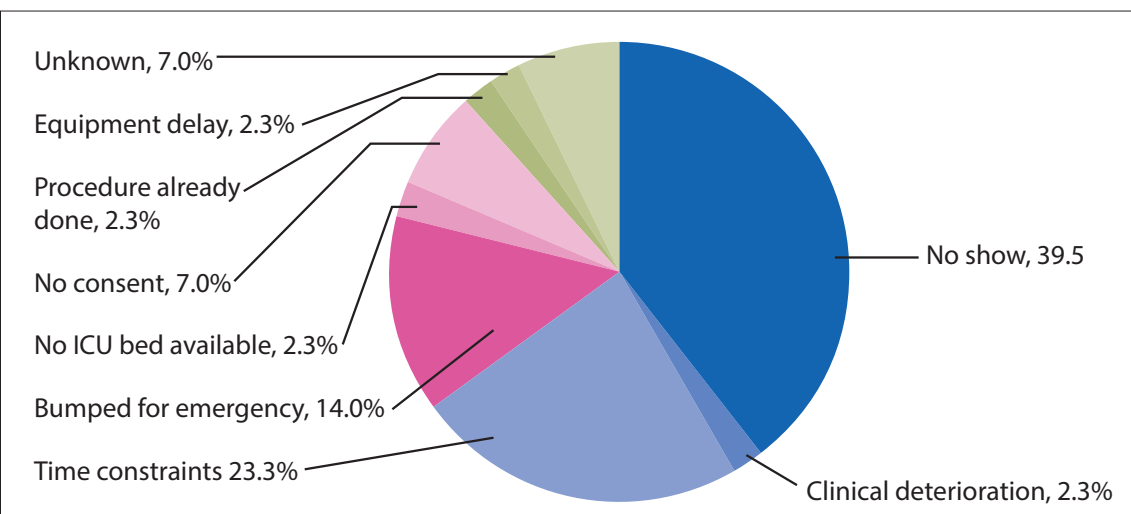

Fig. 1a. Reasons for surgeries being cancelled at the paediatric theatre complex of Chris Hani Baragwanath Academic Hospital, Johannesburg.

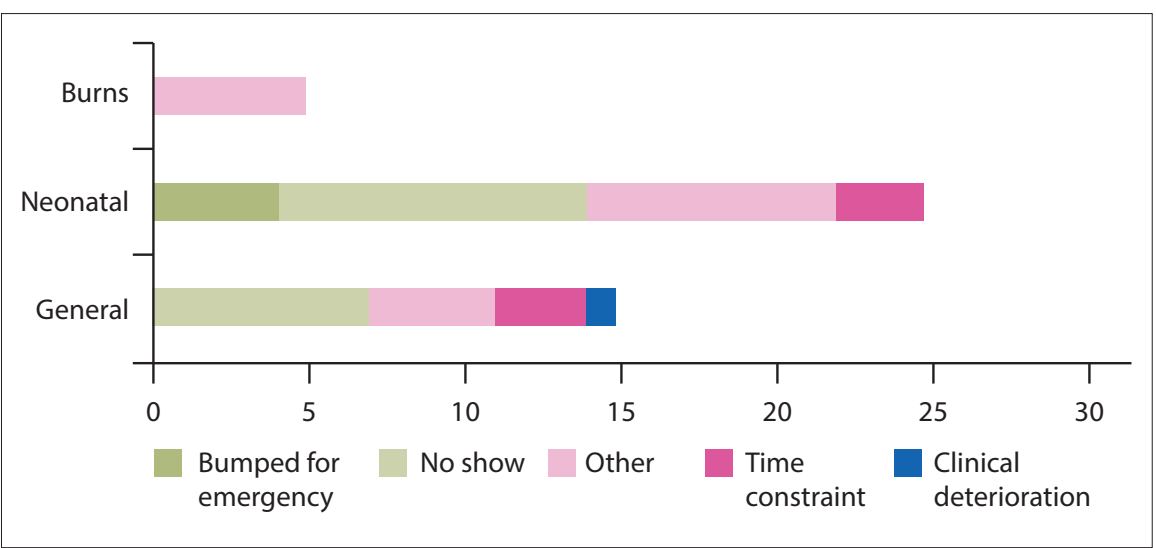

Fig. 1b. Breakdown of reasons for cancellations per theatre. 


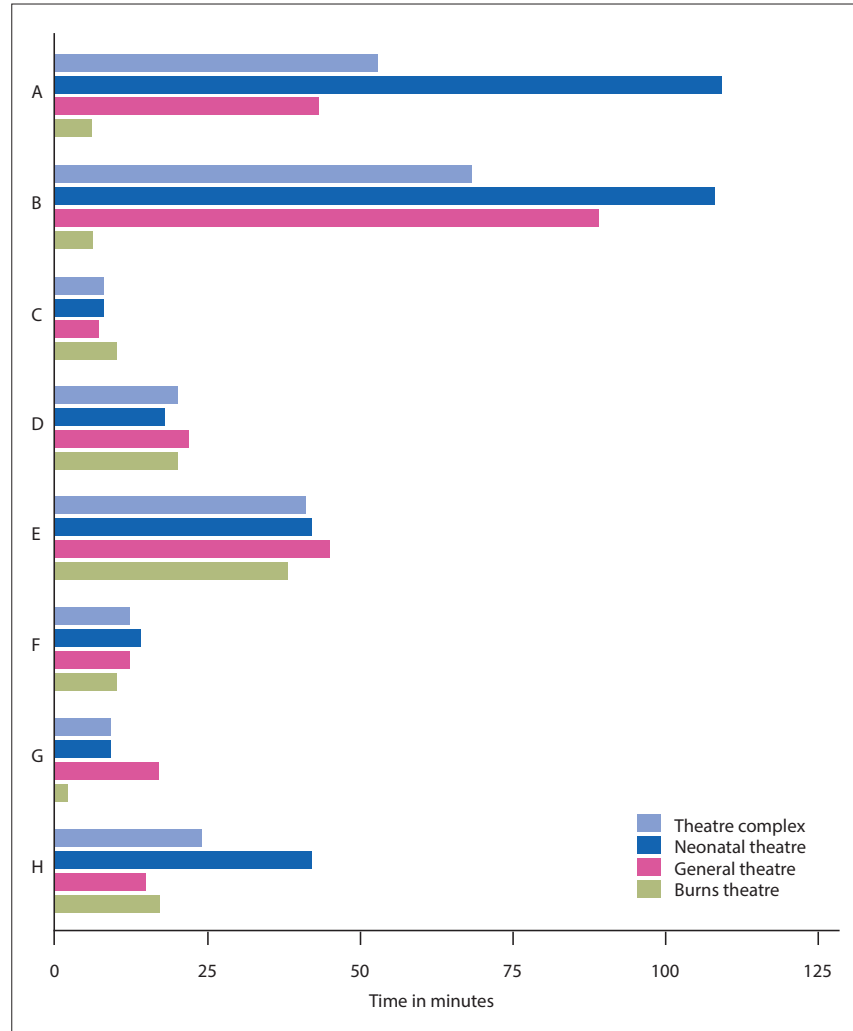

Fig. 2. Average time per task in the respective theatres and the complex combined. A: Time from calling patient to arrival at theatre reception; $B$ : Time from arrival at theatre reception to patient arriving in theatre; $C$ : Time from arrival in theatre to anaesthetic induction; D: Time from anaesthetic induction to start of surgery; E: Time from start of surgery to end; F: Time from end of surgery to patient leaving theatre; G: Time from patient leaving theatre to arriving back in ward; H: Time from previous patient leaving theatre to arrival of next.

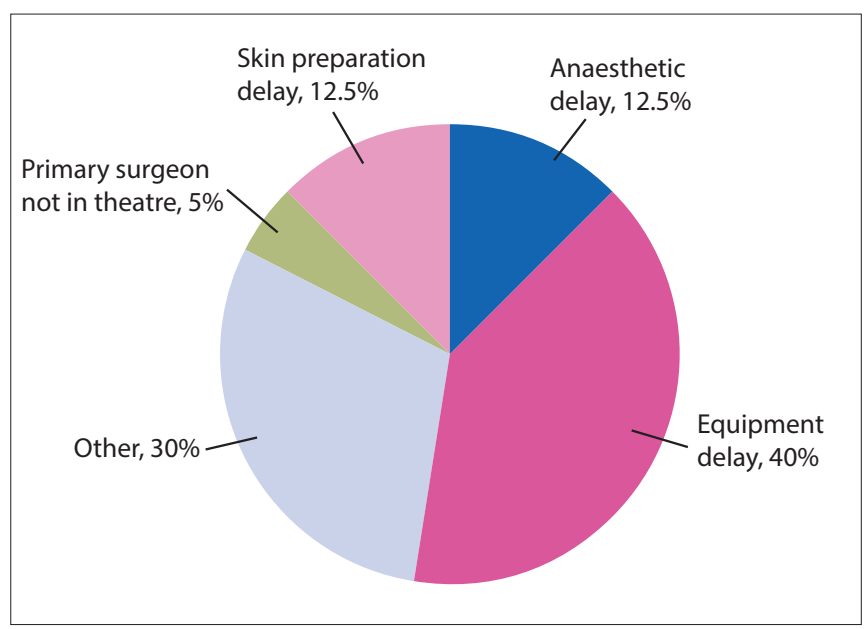

Fig. 3. Reasons for delay between anaesthetic induction and surgical start.

theatres were $62.7 \%$ for the neonatal theatre, $58.2 \%$ for the general theatre and $57.0 \%$ for the burns theatre.

The primary factors contributing to under-utilisation were delays in getting the patient from the ward to theatre reception and delays between anaesthetic induction and the start of surgery (Fig. 3).

Efficiency across the three theatres was calculated as $45.05 \%$, with the lowest efficiency seen at the neonatal theatre $(40.29 \%)$ and the highest in the burns theatre (50.04\%). Efficiency at the general theatre was $47.75 \%$.

\section{Discussion}

The data collected in this study yielded some unexpected results. Ideal theatre utilisation is considered to be approximately $80 \%$, and although the utilisation in the current analysis fell below this, it was higher than anticipated. Overall utilisation for the paediatric theatre complex was $59.8 \%$. This was similar to values in the USA (according to Lobelo ${ }^{[2]}$ ) but notably higher than values recorded in other SA studies, with utilisation rates from $3.2 \%$ in the public setting to $52 \%$ in private settings. ${ }^{[2,4]}$

Despite the utilisation rates of all three theatres being higher than expected, and higher than what has been observed in other SA studies, there is still a considerable backlog for elective surgeries at $\mathrm{CHBAH}$. In addition, cancellations were most often ascribed to time constraints $(23 \%)$, yet most theatres closed before the official closing time.

We propose that 'theatre utilisation' and 'efficiency' should be considered in parallel to understand the results. Efficiency can be defined as the completion of all scheduled cases without any underor overrun. In our study, efficiency was below 50\% (calculated as $45.05 \%$ across all three theatres) and, on days with many cases, this could drop to as low as $6 \%$. Yet utilisation rates were higher than expected. This points to a logistical disconnect, which may be related to several factors.

CHBAH serves a large population, with referrals for elective cases coming from across the Gauteng and North West provinces. This results in a large number of pending cases purely because of the high demand having to be handled by a facility with a finite number of theatre lists per week. In an attempt to accommodate these numbers, theatre lists are often 'overbooked', to take advantage of possible no-shows or other cancellations. So, despite acceptable turnover times, a high number of surgeries had to be cancelled because of 'time constraints' on days when all booked patients arrived for theatre. In addition, cases on the scheduled list would typically be cancelled to accommodate emergency cases that had to be handled in these theatres. As a result, the available time for elective surgeries was reduced, thereby decreasing efficiency but conversely still allowing for high utilisation of the theatres.

Another factor that contributed to high utilisation rates is the time between anaesthetic induction and start of the procedure. This time was longer than expected in $37 \%$ of surgeries, and in half of these cases the delays were due to equipment malfunctions. The delays therefore did not affect utilisation in our analysis, yet markedly affected efficiency. When emergency surgeries and the time between anaesthetic induction and surgical start are taken into account, the overall utilisation dropped to $33.76 \%$, a value more in line with what has been reported previously for SA settings. ${ }^{[1]}$

Cancellations are cited in the literature as negatively affecting theatre utilisation and efficiency. ${ }^{[3]}$ This was also seen in the current study. Lists with more cancellations were associated with poorer utilisation and efficiency values. This suggests that introducing measures to reduce the number of cancellations could improve the efficiency of the operating theatres. Only seven of the 27 lists reviewed over the data collection period were completed; at least one cancellation was recorded in the remaining 20 .

As seen in Fig. 1A, the most common reasons for cancellations were patients not showing up (39.5\%), time constraints (23.3\%) and cases being bumped off the list to accommodate an emergency (14\%). In cases of patients not arriving, theatres were left empty, which reduced both utilisation and efficiency. In cases of time constraints and emergency surgeries, theatres were still being utilised, leading to increased utilisation but decreased efficiency. Improved list scheduling and elective cases being admitted the day 
before the scheduled surgery could help to limit the number of no-shows and so improve both utilisation and efficiency.

Despite theatre lists generally ending before the official theatre closing time, it should be noted that a third $(n=9)$ of the 27 reviewed lists ended late. This resulted in a paradoxical increase in utilisation. These findings suggest that utilisation by itself is not an adequate proxy for operating theatre efficiency. This finding agrees with that from a UK study, which revealed the shortcomings of using utilisation as a measure of efficiency when emergencies and cancellations were not adequately accounted for. ${ }^{[3]}$

Other reasons for delays were analysed in order to identify further areas of improvement. The most notable delay occurred when patients were transferred to the theatre. This included patients not being ready, administrative delays and porters being late. Delays were also noted between a patient's arrival at theatre reception and entering the operating room owing to administrative delays, heater malfunction, and patients not being adequately starved as per anaesthetic protocols. There were no notable delays between a patient's arrival in the operating theatre and anaesthetic induction, despite late arrival of anaesthetists or difficulties with preoperative procedures being noted in some cases. All of these aspects contribute to utilisation rates and are noted as areas for improvement in the administrative context.

Lastly, although the data for these three theatres were combined to provide a general view of utilisation and efficiency of the $\mathrm{CHBAH}$ paediatric theatre complex as a whole, the respective theatres all faced different challenges, related to factors such as the location of the theatre relative to that of the wards and the specific subset of patients the theatre caters for. The interpretation of data drawn from the three theatres is therefore nuanced.

Despite calculated values showing the general and burns theatres theoretically having 'superior' efficiency, they generally completed their lists before 16:00 (14:09 for the general theatre and 15:10 for the burns theatre); standing empty for the remaining time thus decreases utilisation. In contrast, despite the neonatal theatre having the most cancellations $(n=25)$, it also finished its lists last and had the most lists running over time ( $n=5$, compared with two each in the general and burns theatres). This was likely due to greater delays in the neonatal theatre and compounded by it being the primary theatre for emergencies. The neonatal theatre was also used for cases on the general list once. Therefore, despite cancellations and delays, the utilisation rate of this theatre was the highest of the three and its efficiency the lowest $(40.29 \%)$. This is an interesting example of how a theatre can be over-utilised in an inefficient manner, which again points to utilisation being a poor proxy for efficiency. The finding is similar to that of Pandit et al., ${ }^{[3]}$ who noted that gaps between start times of surgeries had very little effect on theatre efficiency and suggested that list scheduling was more important for improving efficiency. ${ }^{[3]}$

The findings of the current study suggest that surgical staff utilise the theatre efficiently, but that administrative issues such as list scheduling and improved systemic access to reduce the number of cancellations could help to increase efficiency.

\section{Conclusion}

At 59.8\%, the paediatric theatre complex at $\mathrm{CHBAH}$ operates below the ideal utilisation rate, although higher than that of surrounding government and private hospitals (and seemingly comparable to utilisation rates in the USA). ${ }^{[2,3]}$ However, low theatre efficiency $(<50 \%)$ observed in this analysis indicates systemic dysfunction. This points to the need for corrective action to improve list scheduling and putting measures in place to limit the number of no-shows and cancellations. Such actions could markedly improve both utilisation and, more importantly, efficiency at $\mathrm{CHBAH}$.

\section{Declaration. None.}

Acknowledgements. The authors thank the staff of the Paediatric Surgery Department at CHBAH for the opportunity to conduct the research.

Author contributions. The manuscript was written jointly by SF, NB, NM, SS, BM, TM and MR. In addition, SF was responsible for final preparation and the submission process. AG, JL and TG supervised and guided the analysis and manuscript preparation.

Funding. None.

Conflicts of interest. None.

1. Hartmann D, Sunjka B. Private theatre utilisation in South Africa: A case study S Afr Med J 2013;103(5):285. https://doi.org/10.7196/samj.6460

2. Lobelo ME. Utilisation of operating theatres at General De La Rey Hospital in the North West Province. Dissertation (Public Health). Johannesburg: University of the Witwatersrand, 2010:1-48.

3. Pandit J, Abbott T, Pandit M, Kapila A, Abraham R. Is 'starting on time' useful (or useless) as a surrogate measure for 'surgical theatre efficiency'? Anaesthesia 2012;67(8):823-832. https://doi.org/10.1111/j.1365-2044.2012.07160.x

4. Ramodise KB. An analysis of theatre utilisation at Sekororo District Hospital. Dissertation (Public Health). Johannesburg, University of the Witwatersrand, 2013:1-67.

Accepted 15 February 2021 